\title{
A Novel Approach of Intuitive K-means Clustering for Renal Calculi Detection in Ultrasound Images
}

\author{
Pawan Kumar Upadhyay ${ }^{1}$, Arun Sharma ${ }^{2}$, and Satish chandra ${ }^{3}$ \\ 1,3 Jaypee Institute of Information Technology, NOIDA, U.P, India \\ 2 State University of New York at Bufflo,New York,USA \\ pawan.upadhyay@jiit.ac.in,arunshar@buffalo.com, satishchandra@jiit.ac.in
}

\begin{abstract}
Medical images are too fuzzy for discrete boundaries. This paper describes a fuzzy rule based seed point optimization in K-mean clustering method with a application in image segmentation. Prior information about the subject helps to elongate the cluster class and able to identify the target seed point smoothly for the detection of renal calculi oftenly called as a kidney stone. Kidney is a source organ for urology disorder which can be protected by efficient kidney stone detection technique in ultrasound images. Proposed method of clustering reduces the number of iterations for elobrating the region of interest in entitled images. This approach promising to give a more accurate solution for ultrasound images and it also enhances the image retrieval as compared to classical clustering methods. The experimental results justify the effectiveness of proposed approach by reducing the computational time without effecting the segmentation quality which can be validated by peak signal to noise ratio value. Results are validated on 150 Ultrasound image samples having six classes of renal calculi.
\end{abstract}

Keywords: K-Means clustering, Fuzzy C-mean, Ultrasound images, Peak Signal to Noise Ratio, Computer Aided Diagnostic system .

\section{Introduction}

In medical image analysis, Ultrasound imaging is one of the most persistent image modalities due to its high adaptability with easy portability in minimum cost.These images are difficult to interpret and require more information for the prognosis of disease in computer aided diagnostic system. Medical image segmentation is known to be difficult due to mottle noises and other erroneous effects. In order to understand these images the first task is to partition it and find out the different objects available in an entitled image. Partitioning a digital image into multiple regions based on pixels is called segmentation. Image segmentation is having many applications in real world senarios. For all the applications, there is no common method which can produce the desired result by considering all the attributes of images like color, noise, brightness, size and shape e.t.c. Most of the image segmentation techniques are based on grayscale image and having some threshold which constitute with similarity and discontinuity. In this method, similarity represents boundedness in an image for cluster region that are akin set of predefined rules. Although, discontinuities are elaborated as a variation in intensities at edges or lines or points.

Image segmentation can be classified into three categories. The first one is unsupervised and it require manual segmentation for its interactiveness, the pixels belonging to the same intensity range are selected manually for one cluster and distinguish with the other set of pixels in available in other cluster of the input image[1]. It requires human supervision, whereas second categary is a combination of supervised and unsupervised segmentation [2]. Third one is intelligent method and support supervised clustering which does not require any human interaction, completely known as automated method for image segmentation[2, 5]. This paper is more prominence over the second method which requires quasi supervision.

Received: February $23^{\text {rd }}, 2016$. Accepted: March $23^{\text {rd }}, 2018$

DOI: 10.15676/ijeei.2018.10.1.9 
Segmentation of ultrasound(US) image is strongly affected by the quality of data. There are certain characteristic of ultrasonic images which make the segmentation task more complex. So, the prior approach for the medical image segmentation is hard clustering [7, 8]. It generally belongs to unsupervised learning method. Two most important benefits of clustering are easy pitching into fuzzy to handle noisy data as well as it has the ability to deal with the data having various dependable variables [8]. The technique examines a collection of points and groups them into clusters based on their similarity index. The similarity measure which are based on distance metric for the substrate of class discrimination $[9,11]$.

Pixels having similar features belong to single cluster and vice versa. Due to this clustering becomes an important data mining techniques which are used for pattern recognition, data analysis, image processing, and many others. Once the features as vector points are extracted and clubbed together into different clusters based upon class attributes of an object in input image. Clustering is having two common classes and one of them is a class called as hard clustering method. K-means clustering algorithm belongs to this class of clustering and having sharp boundaries $[1,15]$. Second class of clustering is soft clustering method like as fuzzy clustering in which data as an image partition into region based on logical representation goven by objective function. Method of clustering slightly turn around towards logical clustering from traditional hard clustering methods as per the requirement of application. Clustering is unsupervised method, optimization in hard clustering will give computational fast results when applied on certain application as compare to logical or soft clustering classical method . Based on this concept, a similar kind of approach is proposed in this paper called as Intuitive K-mean.

In real time applications, one of the important job in image analysis is exact pixel predict and on that basis classifying the exact disease classes in computer aided diagnostic system. When there are no crisp boundaries between the various objects in an image then the fuzzy clustering techniques are used to deal with this difficulty [9]. Otherside of soft fuzzy technique is a particular pixel can belong to one or more cluster due to its degree of membership function value. This method of clustering helps to classify the pixel values more accurately with greater extent. The advantage of fuzzy clustering is that they are detect noisy region or vague boundries in an image very efficiently and help to partition the abnormal region in the data-space efficiently[2, 17]. Unsupervised techniques include all the density based algorithms like as Fuzzy C-means $[4,5,10]$. FCM is the most reliable and commonly used method as it can protect and preserve more information and details as compare to other available techniques. Various density base clustering methods are used in medical image segmentation such as DBSCAN and hierarchical clustering.

\section{Related Work}

Ultrasound images of various frequencies are process by various updated tool and it helps to improvise non- invasive kidney stones detection by effective visualization, but finding the multiple renal calculi is still requires advance software support for ultrasound machine instead of using radiograph or CT imaging [13]. The study has focused on the detection of renal calculi using non-invasive methods of image clustering during segmentation. There are various standard methods for the stone detection which are based on the size of renal calculi visualize in ultrasound imaging [15]. Kidney stones are developed when certain substances like calcium oxalate or other uric acid crystallizes in the urine[13]. These minerals and salt constitute to form crystals, which recursively join together and form a kidney stone. Each type of kidney stone develop with different reason. The generation of these stones in any anatomical region may be caused by inherited factors also and depends upon age, and environmental factors as well [2]. Stones are classified according to their chemical composition and in an image by its characterstic property. The formation of stones may be caused by dietary and lifestyle factors and also acquired metabolic defects leading to crystal formation and growth of kidney stone [13]. Sridhar et al [14] have developed an automated system to detect the renal calculi based on its physical characteristics. An algorithm has been developed to identify the renal calculi using its shadowing effects. The properties of calculi such as size, shape and location have also been extracted. Benoti 
et al proposed a region growing algorithm for segmenting the kidney stones from anatomical regions in a image with certain measures like as Precision, Recall and Yasnoff Measure [14]. Sriparna Saha and Sanghmitra Bandhopadhyay [8] introduced a updated version of Fuzzy Cmeans clustering for medical image segmentation. Fuzzy C-mean is appropiate technique for evaluating the most precise spatial information from an input images but it is slow in computation. In this paper, an algorithm is proposed to increase the robustness and computational speed of the segmentation process.

\section{Clustering Methods for Medical Image Segmentation}

Ultrasound image segmentation is having so many challenges with certain invasive obstacles [15]. Because of the existence of dapple in these images and their deficient contrast, and use of common methods for segmentation is not feasible.

The aim of segmentation is to simplify the interpretation of an image into something that is more significant and effortless to examine [1]. An image can contain several objects so to divide it in compliance with the extracted features, deriving relevant results from it possesses a great challenge. Noise in input image degrades the segmentation process, therefore it must be removed initially by filtering process [2]. One of the best methods to attain segmentation is clustering which can deal with unlabeled data having an unstructured behavior of feature vector [9]. Clustering is a process of arranging objects into groups whose members are same in some way [1]. A cluster is therefore a group of objects that are "similar" in attributes but between them there are objects which are "dissimilar" or not supporting an association of previous object are to be kept in other clusters [9].

Clustering techniques having a taxanomy as supervised clustering which demands human interaction to decide the clustering criteria and the unsupervised clustering which decides the clustering criteria by itself. Supervised clustering has hierarchical approaches and unsupervised clustering has density based clustering methods. These clustering techniques are applied for performing image segmentation [7].

\section{A. K-Means Clustering}

$\mathrm{K}$-means is a repetitious algorithm that calculates mean of each cluster and assigns pixels according to the euclidean distance measure. K-means algorithm is a clustering technique that partitions $M$ pixels into $Z$ clusters, where $Z<M$ is describes in equation[1].

This technique categorizes pixels in an image into $\mathrm{K}$ number of clusters, where $\mathrm{K}$ is a positive integer, on basis of some similarity feature like gray level intensity of pixels and distance of pixel intensities from its centroid. The clusters should not have overlapping with one another. The dimension for the centroid is the same as those of data vectors. Every pixel that is assigned to some clusters is on the basis of their closeness measured according to the euclidian distances. The main benefit of using this clustering algorithm is its simplicity and its computational cost based on application data [2]. They allow it to run efficiently on large data where as the main drawback is that the $\mathrm{Z}$ number of clusters must be determined beforehand. This algorithm does not yield the same result whenever it executes more than one time for same set of input. The classical results obtained as clusters are also dependent on the initial assignments of centroids and subjective measures as well.

\section{K-mean Clustering Algorithm:}

Input: US images with the parameters: number of cluster, iteration number

Output: Segmented image validated on these measures: PSNR, Accuracy

- Convert US image into grayscale image

- Number of cluster : 3

- Assign centroid values to each cluster (1:no_of_cluster).*max ((vector))/(no_of_cluster+1)

- Find distance between selected seed and target seed

- Iteration undergoes for minimum distance with target seed of selected seed. 
Iter_num $=$ MAX $_{1}$

In this next section, Soft k-mean algorithm which is special case of k-mean is discussed in detail. These are the highlighted points which encourage to proceed for the next section and represents the softness in the rigid k-means algorithm with the follwing steps:

- Crisp K-mean is goning to transform into soft K-mean by membership function $(\mu)$.

- Softness increases the segmentation accuracy of noisy image as well as its computation time is as shown in the Table[1].

- Weighted centroid approach generates soft k-mean or fuzzy c-mean method of clustering is more accurate but time consuming as discussed in next section.

\section{B. Fuzzy Clustering}

Fuzzy C-mean clustering is perform by assigning a membership function to each data point corresponding to each cluster center on the basis of distance metrics between data point and cluster center. The degree of membership proven the closness of data point to the center. It is clear that the membership and cluster center are updated according to the objective function in Equation[3,7]. However, some data points may belong to more than one cluster due to fuzzification in the clustering method. As compare to $\mathrm{K}$-means, fuzzy $\mathrm{C}$ - means perform well for overlapped data set. The detail algorithm describes that the random selection of seed point increases the computation speed.

Fuzzy C-means Clustering Algorithm:

Fuzzy C-means (FCM) is clustering technique which allows a section of data belong to two or more than two clusters unlike to have hard partitioning methods and it generates fuzzy C-mean method. In this algorithm a particular pixel can belong to multiple clusters where their degrees of membership may vary.

These are the following steps to detect the stone region in the entitled image of ultrasound (US) as suggested by medical expert.

- Convert the coloured US image to grayscale and compare every pixel with value of cluster center.

- It assigns a value between 0 and 1 describing "how much part of this pixel belongs to each cluster". The membership value of a pixel in a clusters must be 1 for abnormality region.

- Higher the membership value, the more likely the pixel is to belong to that cluster.

FCM optimization is to minimize this objective function describe below

$$
\mathrm{J}=\sum_{i=1}^{n}\left(\sum_{k=1}^{c} \mu_{\mathrm{ik}}^{2}\right)\left|\mathrm{p}_{\mathrm{i}}-\mathrm{c}_{\mathrm{k}}\right|^{2}
$$

Where,

$\mathrm{n}$ : Number of pixels in image,

c: Number of clusters,

$\mu_{\mathrm{ik}}$ : Fuzzy Membership Value of $\mathrm{i}^{\text {th }}$ pixel in $\mathrm{k}^{\text {th }}$ cluster

$p_{i}: i^{\text {th }}$ pixel in the set,

$\mathrm{c}_{\mathrm{k}}$ : Centroid of $\mathrm{k}^{\text {th }}$ cluster,

$\left|\mathrm{p}_{\mathrm{i}}-\mathrm{c}_{\mathrm{k}}\right|$ : Eucledian Distance between $\mathrm{p}_{\mathrm{i}}$ and $\mathrm{c}_{\mathrm{k}}$ defined as

$$
\left|\mathrm{p}_{\mathrm{i}}-\mathrm{c}_{\mathrm{k}}\right|=\sqrt{\sum_{i=1}^{n}\left(\boldsymbol{p}_{\mathrm{i}}-\mathrm{c}_{\mathrm{k}}\right)^{2}}
$$

Centroid of $\mathrm{k}^{\text {th }}$ cluster is calculated by

$$
\mathrm{c}_{\mathrm{k}}=\frac{x}{y} \text { Where } \mathrm{x}=\sum_{\boldsymbol{i}=\mathbf{1}}^{n} \boldsymbol{\mu}_{\mathrm{ik}}{ }^{\mathrm{m}} \mathrm{p}_{\mathrm{i}} \& \mathrm{y}=\sum_{\boldsymbol{i}=\mathbf{1}}^{n} \boldsymbol{\mu}_{\mathrm{ik}}{ }^{\mathrm{m}}
$$

Where, m: Fuzziness Factor

Calculation of Fuzzy membership function is

$$
\mu_{\mathrm{ik}}=1 / \sum_{i=\mathbf{1}}^{\boldsymbol{c}}\left(\frac{|\boldsymbol{p}-\boldsymbol{c}|}{|\boldsymbol{p}-\boldsymbol{c}|}\right)^{2 / \mathrm{m}-1}
$$

Applying Fuzzy C-mean algorithm on US images of kidney stone which is given below. 
Fuzzy C-means Clustering Algorithm:

Input: US images with the parameters: number of clusters, threshold score

Output: Segmented Image validated on these measures:PSNR, Accuracy

- Convert coloured US image into grayscale images.

- Initialize number of cluster : 3 .

- Assign centroid values to each clusters with confined rule of objective function $\mathrm{J}=\sum_{i=1}^{n}\left(\sum_{k=1}^{c} \boldsymbol{\mu}_{\mathrm{ik}}^{2}\right)\left|\mathrm{p}_{\mathrm{i}}-\mathrm{c}_{\mathrm{k}}\right|^{2}$

- Evaluate the euclidian distance between each target centroid and the selected centroid by distance measure

$\left|\mathrm{p}_{\mathrm{i}}-\mathrm{c}_{\mathrm{k}}\right|=\sqrt{\sum_{i=1}^{n}\left(\boldsymbol{p}_{\mathrm{i}}-\mathrm{c}_{\mathrm{k}}\right)^{2}}$

- Evaluate the membership function for each cluster and iterated untill it reaches approximate to target centroid by following two previous steps.

- Update value of objective function defuzzification and segmentation.

Iter_num $=\mathrm{MAX}_{2}$

Initially the number of clusters as $\mathrm{c}(2 \leq \mathrm{c}<\mathrm{n})$ as fuzzification factor (m) and threshold (U) are used defined parameters. All the user-defined input parameters are inculcated and generate the desired output. The PSNR (Peak Signal to Noise Ratio) measures the compactness of the output cluster and various other measures are used to calculate the accuracy of the system. If the parameter is acceptable, then it moves to next step or the parameters are changed and process is repeated undergoes until we get the Least Square Estimated (LSE) distance. This error should be minimized by iterative process and generate segemented image. Although FCM is an successful clustering technique but the consequent membership values does not always correspond to the fine degree of belonging of the data, and it may be inaccurate in noisy images .By considering this issue, we adopt the closely related measure as peak signal to noise ration of image for the all the three methods discuss in this paper. Our objective is reduce the number of iteration and enhances the computational speed of classical clustering methods( as discussed in previous and in this scetion). So, we proposed an algorithm based on the hybrid approach of K-means and Fuzzy C-means clustering in the next. Proposed algorithm is computational efficient and robust as compare to existing algorithms.

There are certain gaps in the non-Fuzzy K-means and Fuzzy C-means clustering methods. These shortcoming of classical methods are highlighted with the following points :

- Hard clustering methods such K-means clustering hard boundaries between the clusters find difficulty in seperating the exact region of interest from background image.

- Fuzzification in K-means clustering increases its softness due to its membership function.

- The added property of vagueness extend the boundaries of clusters to some extent for improving the renal calculi detection in US images but it is computationally slow in execution

To improve the speed and accuracy of clustering methods for image segmentation, a hybrid approach of clustering is proposed in the next section.

\section{Proposed : Intuitive K-mean Clustering}

Proposed clustering technique is based on the hybridization of K-means and Fuzzy C-means classical clustering techniques. The K-Means clustering is a distance based clustering method. In addition to this, fuzzy rule are incorporate to the standard K-means clustering for improving the its level for performing acitivity in image space and detect the soft target seed point at certain threshold value with more accuracy. According to the objective function as shown in equation[10,11] are used to clustered the various regions of image in minimum number of iterations. Novel technique is more robust and computational efficient as compared to existing methods which are used to detect the renal calculi in US images. Unsupervised clustering methods produces centroid of label classes based on their attributes. Accuracy of the new 
clustering algorithm depends on seed selection which is having the minimum distance with target seed. Updating the seed selection with rule base in K-Means generates Intuitive K-means.

There are certain reasons to propose a intuitive method for the detection of renal calculi in US images are describes in the following points :

In $\mathrm{K}$-mean clustering, objective function consider the intra-class similarity . The vagueness in the boundary of ROI due to shadowing of renal calculi, they are not discriminated with the exact boundaries of abnormal region.Due to this reason, shadowing which are interpreted as actual calculi, increases the mean square error(MSE) and reduces the PSNR of segmented image.

- Fuzzy C-mean clustering added vagueness due to its membership function and few pixels of shadowing region are kept commonly as in both clusters of object and background.To improve the fuzzy cluster points in a constrainted boundry, we requires a seed point elongation for detecting the exact boundaries of kidney stone region as a cluster from its background image.

- Shadowing of renal calculi and calcium based anatomical region pretains as abnormality and having lower value of pixel than the average value in US images. This can be detected by the new objective function as shown in equation[10,11] of the proposed method. However, the updated objective function work on boundary values [as shown in Equation11] and help to minimize the number of iteration .

- The new approach of clustering is having a quasi hard property which adopt combine features of K-means as well as fuzzy c-means clustering.

\section{Intuitive $K$-mean algorithm:}

Input: US images with the parameters: number of clusters, threshold score

Output: Segmented Image validated on these measures:PSNR, Accuracy

- Read the coloured US image and convert to gray scale

- Number of cluster : 3

- Generate the normalized gray scale values and generate the mean of this vector as centroids.

- Assign centroid values to each clusters with the optimized objective function in equation [10]

Objective Function

$$
\mathrm{J}=\sum_{i=1}^{n}\left(\sum_{k=1}^{c} \mu_{\mathrm{ik}}^{2}\right) *[\max (\mathrm{Vi})-\operatorname{mean}(\mathrm{Vi})] / \mathrm{n}+1
$$

- Mean vector computation by the improved objective function for seed selection having minimum distance with target seed of the cluster.

Mean vector (MV)e:

$$
\begin{aligned}
& \sum_{i=1}^{n} C i *[\max (V i)-\operatorname{mean}(\mathrm{Vi})] / n+1 \\
& n=\text { no of clusters, } C i=\text { cluster having } \mathrm{i}^{\text {th }} \text { number of pixels } \\
& V i=\text { Gray value of an pixel in a image- min gray value }+1
\end{aligned}
$$

- Updation of objective function using distance metrics will reduces the computation time.

- Update the value of objective function for image segmentation in minimum iteration in previous step is describes in equation number $[10,11]$.

Iter_num $=\mathrm{MAX}_{3}$

Equation[10,11], represents the difference between maximum pixels intensity of an image with mean pixel intensities of an renal calculi. The distribution of pixels in between the two values are seems to a Gaussian and give elongated seed point. The obtained PSNR among the two boundary values are maximum with most appropriate region of interest as renal calculi .Robustness of the proposed algorithm are validated by standard measures like as PSNR. Number of iteration and its computation time are inversely proportional to each other, when applied on US images to detect the renal calculi.

Comparison between the maximum iteration between all three clustering methods are describe below :

$$
\mathrm{MAX}_{2}>\mathrm{MAX}_{1}>\mathrm{MAX}_{3}
$$


Proposed algorithm is having minimum number of iterations as describe in $\mathrm{MAX}_{3}$. The iterations among different classes of renal calculi are compared in the experimental results as shown in Figure[1].

\section{Experimental Results}

Six classes of renal calculi varying from $2-5 \mathrm{~mm}$ in different anatomical region are efficiently detected by proposed method of clustering. The measure of PSNR validate the accuracy in segmented calculi as region of interest from the input image. The related dataset of ultrasound images are obtained from various sources [19,21]. The results drived from 150 ultrasound images having 25 images per class. The region of Interest is obtained from the scaled image of $256 \times 256$ after prepossessing the original images.

The platform used is Matlab (Version 2015a) with Intel i5 processor (3.2GHZ, 6GB RAM). US Ground truth images of various classes are described below with resultant images after applying the proposed method.

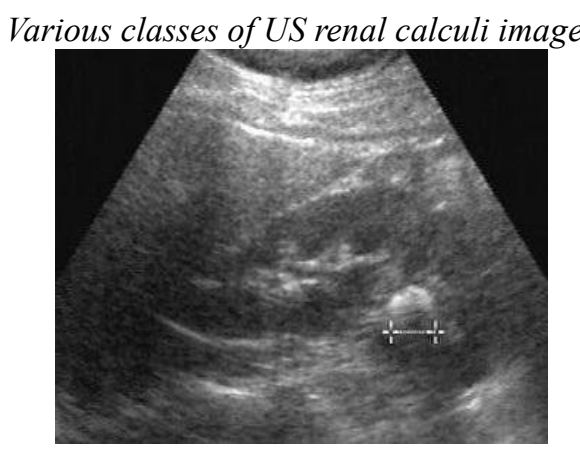

Class 1: Renal Calculi

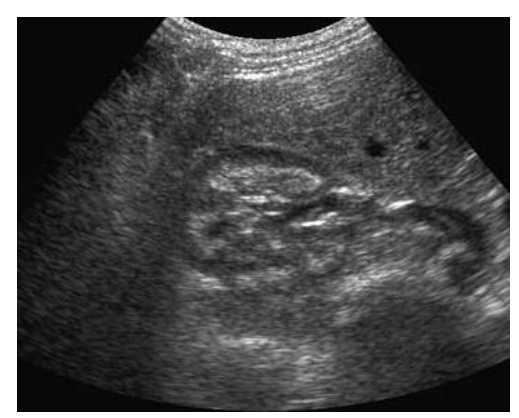

Class 2: Staghorn Calculus

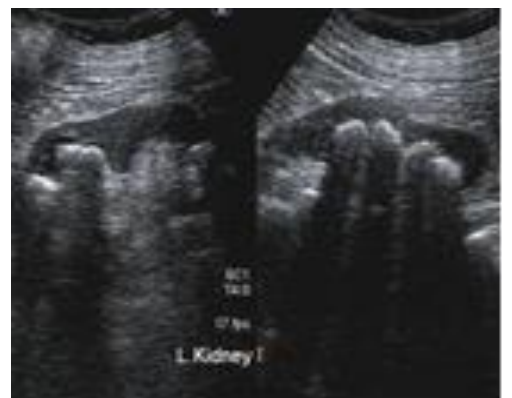

Class 3: Multiple Renal Calculi 
Pawan Kumar Upadhyay, et al.

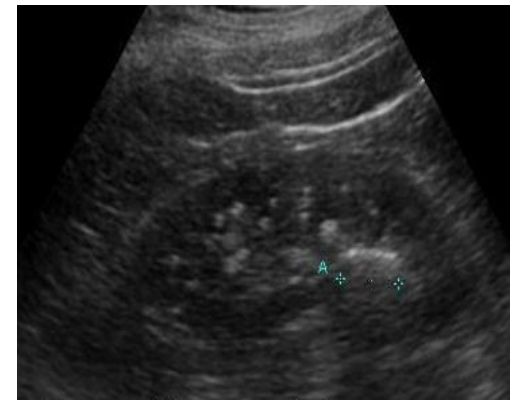

Class 4 : Small Multiple

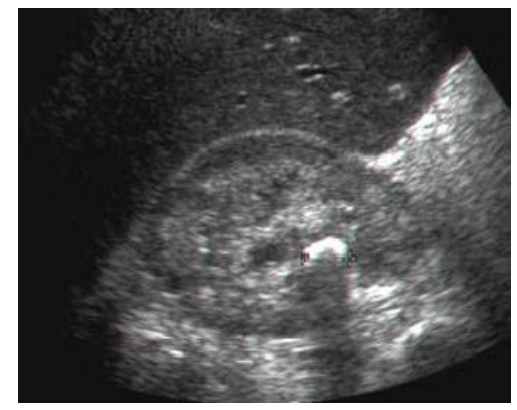

Class 5: Nephrocalcinosis

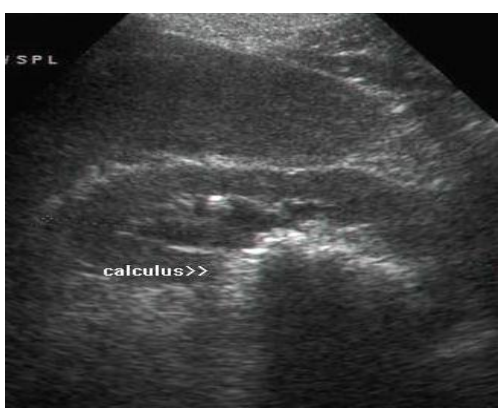

Class 6: Anderson-Carr Renal Calculi Kidney

Segmentation US images using proposed method

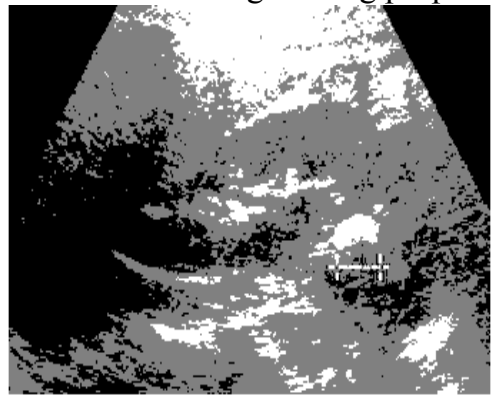

Class 1: Renal Calculi 


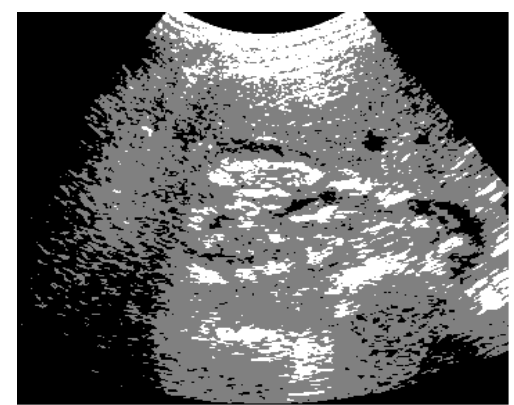

Class 2 : Staghorn Calculus

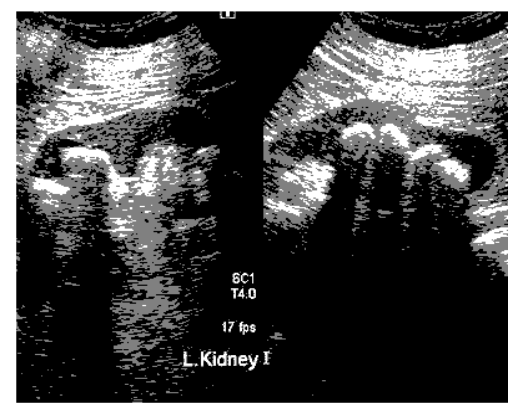

Class 3: Multiple Renal

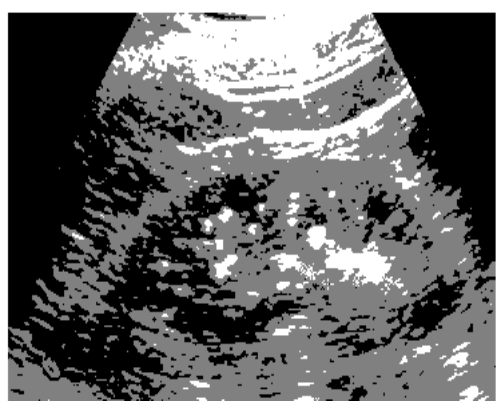

Class 4: Small Multiple Renal Calculi

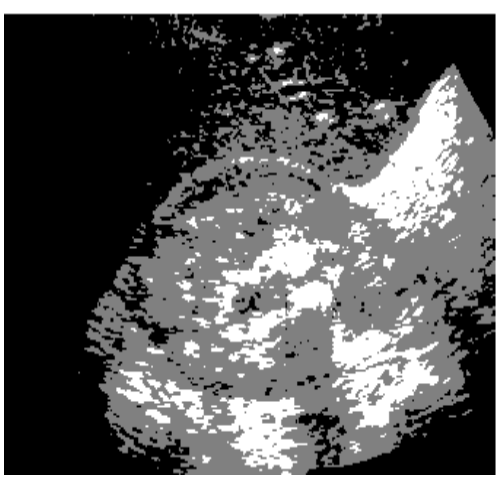

Class 5: Nephrocalcinosis 


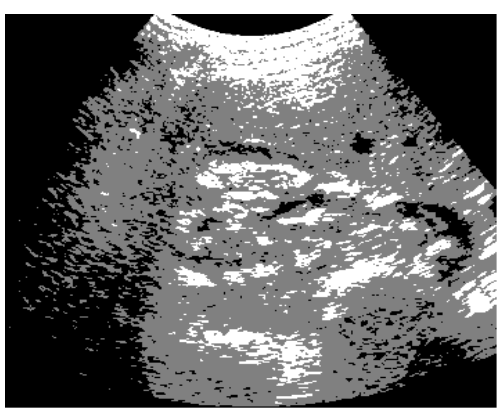

Class 6: Anderson-Carr

Kidney Calculi

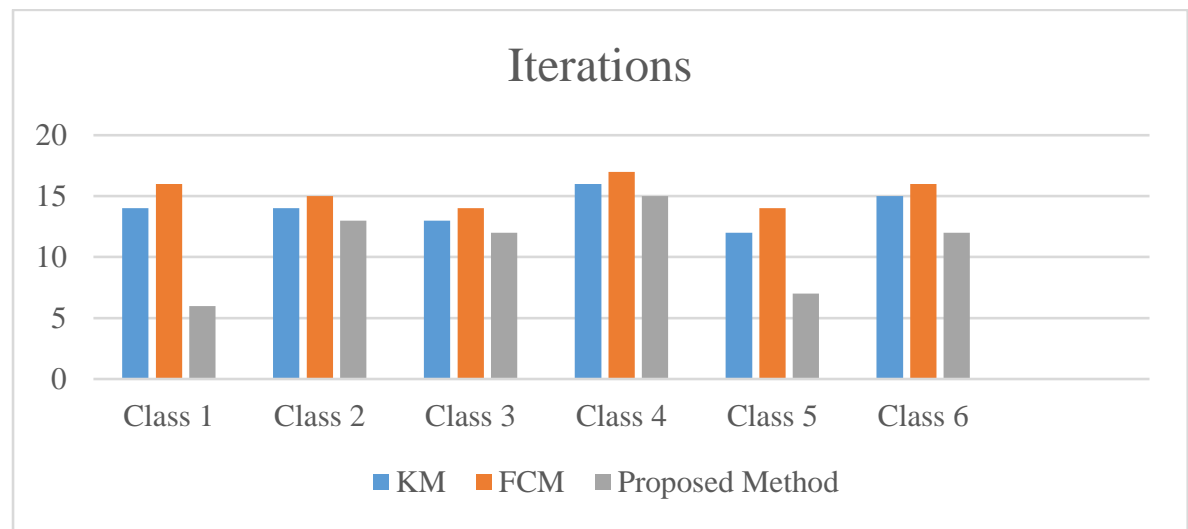

Figure 1. No of Iteration vs Classes of renal calculi

Comparison of proposed method with other clustering techniques are described in the above Figure 1. Proposed method requires minimum iterations for detecing each of renal calculi in ultrasound images.

The number of iteration is marked with $\left(\mathrm{MAX}_{3}\right)$ as compared to $\mathrm{FCM}\left(\mathrm{MAX}_{2}\right)$ and $\mathrm{KM}$ $\left(\mathrm{MAX}_{1}\right)$. Hence the result are investigated from the Equation 13 and its computational complexity is decribes in the Table[1].

Table 1. Comparision of computaion complexity of cluster algorithms

\begin{tabular}{|c|c|}
\hline Algorithm & Time Complexity \\
\hline K means & $\mathrm{O}(\mathrm{ncd})$ \\
\hline Fuzzy C mean & $\mathrm{O}(\mathrm{ncmd})$ \\
\hline Proposed & $\mathrm{O}(\mathrm{nc})$ \\
\hline
\end{tabular}

Where, $\mathrm{n}$ : Number of pixels in image, c: Number of clusters,m: membership function cost $\mathrm{d}$ : distance matrix calculation cost.

Intutive K-mean having the bounded distance from maximum intensity of pixels to mean intensity of ROI and it help to reduce the number of iterations. The comparison of computation time taken by proposed technique as compare to classical clustering techniques as K-means and Fuzzy C-means on a standard image atlas of renal calculi is shown in the Table[1]. 
Table 1. Time chart of clustering algorithms

\begin{tabular}{|l|c|c|c|}
\hline \multicolumn{1}{|c|}{ Methods } & K-means & $\begin{array}{c}\text { Fuzzy } \\
\text { C-means }\end{array}$ & Proposed Method \\
\hline Renal Calculi & 0.912 & 1.022 & 0.612 \\
\hline Staghorn Calculus & 1.502 & 1.534 & 1.470 \\
\hline Multiple Renal Calculi & 1.333 & 1.483 & 1.249 \\
\hline Small Multiple Renal Calculi & 1.108 & 1.561 & 1.010 \\
\hline Nephrocalcinosis & 0.931 & 1.027 & 0.652 \\
\hline Anderson-Carr Kidney & 1.617 & 1.664 & 1.291 \\
\hline
\end{tabular}

Peak Signal to Noise Ratio(PSNR): It is the ratio between maximum possible signal strength and noise level that affects the representation of output image. PSNR is usually expressed in unit of decibel, also it is commonly used as an evaluating measure for quality assesment of an image. The signal in this case is entitled image pixels data and the noise is the error during acquisition and restoration. High value of PSNR indicates the high quality of image.

$$
P S N R=20 \log _{10}\left(\frac{M A X_{f}}{\sqrt{M S E}}\right)
$$

Here, $M A X_{f}$ is the maximum possible pixel value of the entitled image and MSE (Mean Square Error) can be defined as:

$$
M S E=\frac{1}{m n} \sum_{0}^{m-1} \sum_{0}^{n-1}\|f(i, j)-g(i, j)\|^{2}
$$

Where $\mathrm{f}$ is matrix data of input image, $\mathrm{g}$ is matrix data of degraded image, $\mathrm{m}$ : rows of pixels, $\mathrm{n}$ : columns of pixels.

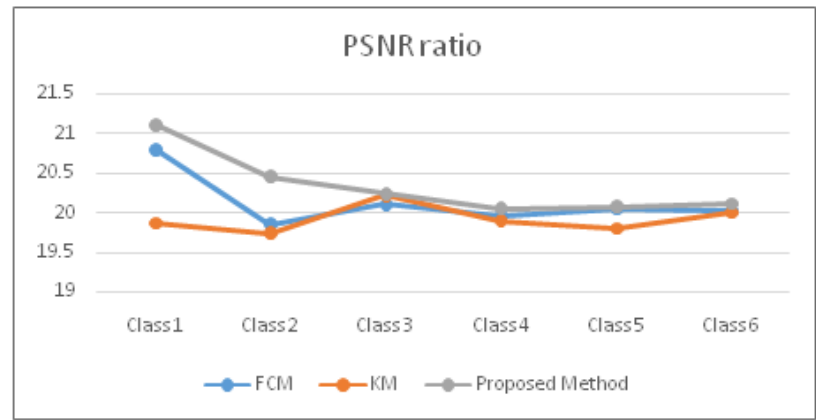

Figure 2. Peak Signal to Noise Ratio vs Classes of Renal Calculi

and the related sample of multiple renal calculi are shown below which decribes the value of PSNR in deceasing order as illustrated in Figure 3:(a) Proposed Method:Blue(17.08) (b) FCM: Green(16.68) (c) Kmeans: Red(15.09)

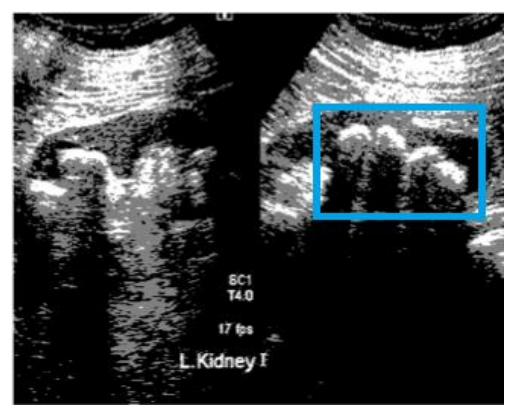

(a) 


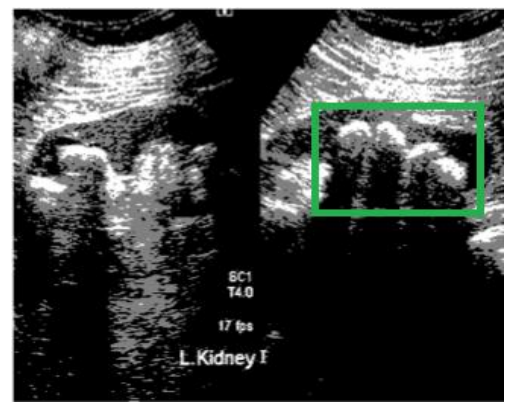

(b)

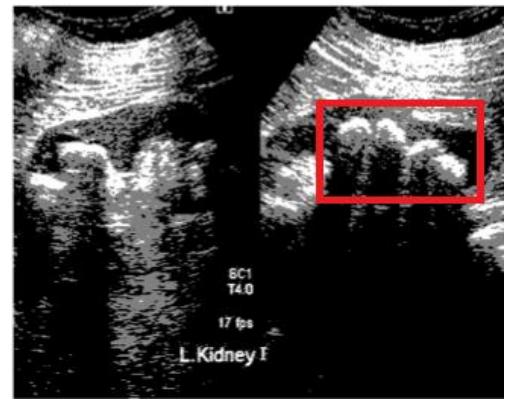

(c)

Figure 3: PSNR value of renal calculi region (ROI) by three algorithms

In the paper, Proposed method of clustering gives highest PSNR value as compared to FCM and K-means. Figure 2 describes the comparison of PSNR ratio amoung various classes of renal calculi and it visualization with actual value for the sample class of multiple renal calculi is illustrated in Figure 3 . The proposed method results, signifies that if the value of MSE is low, better will be the quality of the image and higher will be PSNR.

\section{Conclusion}

This paper presents a logical clustering technique ,based on the concept of seed selection.A new method of intutive K-means, enhances the performance in terms of accuracy as well as reduces the computation time for selection of most appropriate seed point. This seed point is the key source of optimization in classical clustering algorithm. It helps to detect renal calculi of various classes in ultrasound images more efficiently and accurately. Experiments were performed on 150 samples of US images of kidney stone. Robustness of the segmented images are evaluated on standard parameters like as PSNR. The obtained accuracy of segmented image by proposed method is much better than existing algorithms. In future, new method of clustering will going to apply for various other modalities of images used in the field of medical diagnostic.

\section{References}

[1]. Chinki Chandhok, Soni Chaturvedi and Dr.A.A Khurshid, "An Approach to Image Segmentation using K-means Clustering Algorithm", International Journal of Information Technology, Vol.1, Issue. 1,pp.11-17, August 2012.

[2]. P. Sravani and S.Deepa, "A Survey on Image Segmentation Techniques and Clustering", International Journal of Advance Research in Computer Science and Management Studies, Special issue,pp.45-51, December 2013.

[3]. M.Mary Synthuja Jain Preetha, L.Padma Suresh Seeded Region Growing", International Conference on Computing, Electronics and Electrical Technologies, pp.576-583, 2012. 
[4]. Mohit Agarwal and Gaurav Dubey, "Application of clustering technique for Image Segmentation", International Journal of Advanced Research in Computer Science and Software Engineering, Vol.3, issue.4, pp. 764-766, April 2013.

[5]. Soumi Ghosh and Sanjay Kumar Dubey, "Comparative Analysis of K-Means and Fuzzy CMeans Algorithms", International Journal of Advanced Computer Science and Applications, Vol. 4, No.4, pp.35-39, 2013.

[6]. Yong Yang and Shuying Huang, "Image Segmentation by FCM Clustering Algorithm", Computing and Informatics, Vol. 26, pp.17-31, 2007.

[7]. M. Lalitha, M. Kiruthiga and C. Loganathan, "A Survey on Image Segmentation through Clustering Algorithm", International Journal of Science and Research, Vol 2, Issue 2, pp.348-358, February 2013.

[8]. Princy Mishra, Shikha Agarwal and Usha Kiran, "Survey Paper Based On Medical Image Segmentation and Clustering Techniques", International Journal of Advanced Research in Computer Engineering \& Technology, Vol.2, Issue.12, pp.3191-3194, December 2013.

[9]. B.Sathya and R.Manavalan, "Image Segmentation by Clustering Methods: Performance Analysis", International Journal of Computer Applications, Vol. 29- No.11, pp.27-32, September 2011.

[10]. Neelam Kumari, Bhawna Sharma and Dr. Deepti Gaur, "Implementation of posibilistic fuzzy c-means clustering algorithm”, International journal of scientific \& engineering research, Vol.3, Issue.11, pp.1-9, November 2012.

[11]. Md. Iqbal Quraishi, J Pal Choudhury and Mallika De, "Image Recognition and Processing Using Artificial Neural Network", IEEE, 2012.

[12]. Koushal Kumar and Abhishek, "Artificial Neural Networks for Diagnosis of Kidney Stones Disease", I.J. Information Technology and Computer Science, Vol.7, pp.20-25, July 2012.

[13]. P.R.Tamilselvi and P.Thangaraj,"Segmentation of Calculi from ultrasound Kidney Images by Region Indicator with Contour Segmentation Method", Global Journal of Computer Science and Technology, Vol.11, pp 43-51, Dec 2011.

[14]. Jie Wu, Skip Poehlman, Michael D. Noseworthy and Markad V. Kamath, "Texture feature based automated seeded region growing in abdominal MRI segmentation", J. Biomedical Science and Engineering, Vol.2, pp.1-8, 2009.

[15]. Shilpa Kamdi and R.K.Krishna, "Image Segmentation and Region Growing Algorithm", International Journal of Computer Technology and Electronics Engineering, Vol.2, issue.1,pp.103-107,2011.

[16]. F. Masulli, A. Schenone and A.M. Massone, "Fuzzy clustering methods for the segmentation of multimodal medical images",2000

[17]. Saccomani MD, Pizzini C, Piacentini GL, Boner AL, Peroni DG,".Analysis of urinary parameters as risk factors for nephrolithiasis in children with celiac disease" J Urol. 2012 Aug;188(2):566-70. doi: 10.1016/j.juro.2012.04.019.

[18]. http://www.ultrasound-images.com/renal-calculi/. 


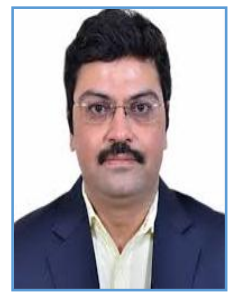

Pawan Kumar Upadhyay is working as an Assistant Professor in the Department of Computer Science and Engineering at Jaypee Institute of Information Technology, Noida, India. His current research interests are Medical Image Analysis, Computer Vision, Machine Learning and High Performance Computing.

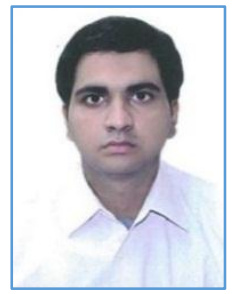

Arun Sharma is pursuing Master of Science in Computer Science from State University of New York at Buffalo, New York, U.S.A. He received his Bachelor and Master of Technology in Computer Science from Gautam Buddha University, Greater Noida, India. His current research interests are Medical Image Analysis, Spatiotemporal Data Mining, Big Data and Machine Learning.

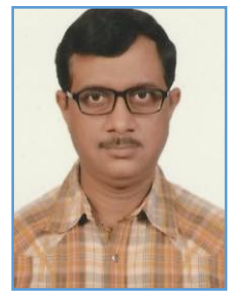

Satish Chandra is working as an Associate Professor in the Department of Computer Science and Engineering at Jaypee Institute of Information Technology, Noida, India. He has completed his Doctorate in Computer Science from Jaypee University of Information Technology, Solan, India. His research area includes Artificial Intelligence, Machine Learning and Metaheuristic computing. He has guided approximately 100 post graduate and undergraduate project thesis. 